\title{
A new class of linearizable equations
}

\author{
R. Hernández Heredero, A. Shabat and V. Sokolov
}

October 24, 2018

\begin{abstract}
Using the symmetry approach, we find a class of integrable nonlinear PDEs with dispersion law $\omega(k)=k^{\frac{3}{2}}$. All these equations turn out to be linearizable by means of a differential parametrization.
\end{abstract}

\section{Introduction}

We consider the problem of classification of PDEs

$$
q_{t t}=q_{x x x}+F\left(q, q_{x}, q_{t}, q_{x x}, q_{t x}\right) .
$$

having higher symmetries. These equations possess an unusual dispersion law $\omega(k)=k^{\frac{3}{2}}$. Moreover one can prove that for such equations there are no higher conserved densities and therefore they are nonhamiltonian.

The first example

$$
q_{t t}=q_{x x x}+3 q_{x} q_{t x}+\left(q_{t}-3 q_{x}^{2}\right) q_{x x}
$$

of such equations has been found in [1]. Particular solutions of (2) feature moving branchpoints singularities. For instance, the simplest self-similar solutions of the form $q=$ $q(x+k t)$ satisfy the ODE

$$
y^{\prime}=y(y-k)^{2}+\text { const }
$$

where $q_{x}=y$. It is obvious that they have singularities of such type.

Nevertheless, equation (2) possesses a degenerate Lax representation which implies infinitely many higher symmetries and corresponding invariant solutions [1]. Three higher symmetries are as follows

$$
\begin{aligned}
& q_{t_{1}}=q_{x x}+2 q_{x} q_{t}-q_{x}^{3} \\
& q_{t_{2}}=q_{x t}+q_{x} q_{x x}+q_{t}^{2}+q_{x}^{2} q_{t}-q_{x}^{4} \\
& q_{t_{3}}=q_{t t}+3 q_{x} q_{t}^{2}-2 q_{x}^{3} q_{t} .
\end{aligned}
$$

The whole hierarchy of symmetries can be reproduced by a recursion operator

$$
\mathcal{R}_{0}=-q_{x}+2 D^{-1} q_{x x}+D^{-1} D_{t}=A+B D_{t}
$$

acting on the seed symmetries $q_{x}$ and $q_{t}$. 
In Section 2 we generalize the main concepts of the symmetry approach, such as the formal recursion operator and canonical conserved densities for the case of non-evolutionary equations of the form

$$
q_{t t}=F\left(q, q_{1}, q_{2}, \ldots, q_{n}, q_{t}, q_{t 1}, q_{t 2}, \ldots, q_{t m}\right) .
$$

Such type of equations were excluded from consideration in works $[2,3$, 4], where only evolution equations were investigated. Obviously, equation (4) can be rewritten as a system of two evolution equations. For example, equation (2) is equivalent to

$$
u_{t}=\left(v_{x}-u v\right)_{x}, \quad v_{t}=\left(u-v^{2}\right)_{x}
$$

where $v=q_{x}$. However the matrix coefficients of leading derivatives for such systems have the structure of a Jordan block, whereas in papers $[5,6,7]$, devoted to systems of evolution equations, the leading matrix was supposed to be diagonalizable.

Using the technique of canonical conserved densities, we find all equations (1) possessing infinitely many higher symmetries. It turns out (see Section 3) that all these equations are related to second order evolution equations

$$
u_{t}=H\left(x, u, u_{x}, u_{x x}\right)
$$

having higher symmetries. It was shown by Sergey Svinolupov [8] that any such equation (5) can by reduced to one of the following equations

$$
\begin{gathered}
u_{t}=u_{x x}+f(x) u, \\
u_{t}=u_{x x}+2 u u_{x}+g(x), \\
u_{t}=\left(\frac{u_{x}}{u^{2}}+\lambda x\right)_{x}, \\
u_{t}=\left(\frac{u_{x}}{u^{2}}+\lambda_{1} x u+\lambda_{2} u\right)_{x}
\end{gathered}
$$

by a contact (or point) transformation

$$
\bar{x}=\phi\left(x, u, u_{x}\right), \quad \bar{u}=\psi\left(x, u, u_{x}\right),
$$

where

$$
\frac{\partial \phi}{\partial u_{x}}\left(\frac{\partial \psi}{\partial u} u_{x}+\frac{\partial \psi}{\partial x}\right)=\frac{\partial \psi}{\partial u_{x}}\left(\frac{\partial \phi}{\partial u} u_{x}+\frac{\partial \phi}{\partial x}\right) .
$$

All these equations can be linearized by simple differential substitutions (see [8]).

In Section 3 we show that all equations (1) from our list admit a parametrization of the form

$$
q_{x}=K\left(q, q_{y}, q_{y y}\right), \quad q_{t}=S\left(q, q_{y}, q_{y y}, q_{y y y}\right),
$$

where $q_{x}=K$ is a linearizable equation of second order and $q_{t}=S$ is a higher symmetry of this equation. For example, the parametrization (7) of equation (2) is given by

$$
q_{x}=\frac{q_{y y}}{q_{y}^{2}}, \quad q_{t}=-\frac{q_{y y y}}{q_{y}^{3}}+3 \frac{q_{y y}^{2}}{q_{y}^{4}} .
$$


Any common solution $q(x, t, y)$ of system (7) gives us an one-parameter family of solutions for the integrable equation (1). However, the general solution of (7) depends on one function $q_{0}(y)=q(0,0, y)$ of one variable, whereas the general solution of (1) depends on two functions of one variable.

Although a general idea of such parametrization is contained in [9, 1], explicit formulas for linearization of equation (2) were obtained first by V. E. Adler.

Acknowledgements. The authors are grateful to V. Adler for useful discussions. This research was partly supported by the Russian Fund for Basic Research (grants 0101-00874-A, 02-01-00431-A and 00-15-96007-L) and Spanish project DGICYT PB98-0821.

\section{Classification of third order equations.}

\subsection{Integrability conditions.}

For equation (4) all mixed derivatives of $q$ containing at least two time differentiation can be expressed in terms of

$$
q, q_{x}, q_{x x}, \ldots, q_{i}, \ldots, \quad q_{t}, q_{t 1}=q_{t x}, q_{t 2}=q_{t x x}, \ldots, q_{t i}, \ldots
$$

in virtue of (4). The derivatives (8) are regarded as independent variables.

An equation

$$
q_{\tau}=G\left(q, q_{1}, q_{2}, \ldots, q_{r}, q_{t}, q_{t 1}, q_{t 2}, \ldots, q_{t s}\right)
$$

compatible with (4) is called infinitesimal (local) symmetry of (4). Compatibility implies that the function $G$ satisfies the equation $\mathcal{F}(G)=0$, where

$$
\mathcal{F}=D_{t}^{2}-\sum_{i=0}^{n} \frac{\partial F}{\partial q_{i}} D_{x}^{i}-\left(\sum_{i=0}^{m} \frac{\partial F}{\partial q_{t i}} D_{x}^{i}\right) D_{t} \stackrel{\text { def }}{=} D_{t}^{2}-\left(M+N D_{t}\right)
$$

is the linearization operator for equation (4).

In order to rewrite consistency conditions of (4) and (9) in terms of a series of conservation laws

$$
\left(\rho_{i}\right)_{t}=\left(\sigma_{i}\right)_{x}
$$

for (4), one can use a formal Lax representation of the problem. The linearization of equations (4), (9) gives rise to the compatibility problem for linear equations

$$
\phi_{t t}=\left(M+N D_{t}\right) \phi, \quad \phi_{\tau}=\left(A+B D_{t}\right) \phi
$$

or, equivalently,

$$
\Phi_{t}=F_{*} \Phi, \quad \Phi_{\tau}=G_{*} \Phi, \quad \Phi=\left(\begin{array}{c}
\phi \\
\phi_{t}
\end{array}\right), \quad F_{*}=\left(\begin{array}{cc}
0 & 1 \\
M & N
\end{array}\right)
$$

where

$$
G_{*}=\left(\begin{array}{cc}
A & B \\
\hat{A} & \hat{B}
\end{array}\right), \quad \hat{A} \stackrel{\text { def }}{=} A_{t}+B M, \quad \hat{B} \stackrel{\text { def }}{=} B_{t}+B N+A
$$


The cross differentiation yields

$$
D_{t}\left(G_{*}\right)=\left[F_{*}, G_{*}\right]+D_{\tau}\left(F_{*}\right)
$$

where $F_{*}, G_{*}$ are matrix differential operators. The crucial step in the symmetry approach (see $[3,5,10]$ and references there) is to consider instead of above equation one as follows

$$
D_{t}(R)=\left[F_{*}, R\right]
$$

where $R$ is matrix pseudo-differential operator. We call $R$ matrix formal recursion operator.

Denoting as before $R_{11}=A, R_{12}=B$ we can rewrite (13) as follows

$$
\begin{gathered}
A_{t t}-N A_{t}+[A, M]+\left(2 B_{t}+[B, N]\right) M+B M_{t}=0 \\
B_{t t}+2 A_{t}+[B, M]+[A, N]+\left([B, N]+2 B_{t}\right) N+B N_{t}-N B_{t}=0 .
\end{gathered}
$$

If $R_{1}, R_{2}$ are formal matrix recursion operators, then $R_{3}=R_{1} R_{2}$ is a formal recursion operator as well and we find using (11) that

$$
A_{3}=A_{1} A_{2}+B_{1} B_{2} M+B_{1} A_{2, t}, \quad B_{3}=A_{1} B_{2}+B_{1} A_{2}+B_{1} B_{2} N+B_{1} B_{2, t} .
$$

Identities (14),(15) mean that the scalar pseudo-differential operator $\mathcal{R}=A+B D_{t}$ is related to the linearization $\mathcal{F}$ of equation (4) by

$$
\mathcal{F}\left(A+B D_{t}\right)=\left(\bar{A}+B D_{t}\right) \mathcal{F},
$$

where $\bar{A}=A+2 B_{t}+[B, N]$. The pseudo-differential operator $\mathcal{R}=A+B D_{t}$, whose components

$$
A=\sum_{-\infty}^{n} a_{i} D_{x}^{i}, \quad B=\sum_{-\infty}^{m} b_{i} D_{x}^{i}
$$

satisfy (14) and (15), is called scalar formal recursion operator for equation (4). If $A$ and $B$ are differential operators (or ratios of differential operators), condition (17) implies the fact that operator $\mathcal{R}$ maps symmetries of equation (4) to symmetries. However we are using the notion of formal recursion operator for a completely different aim.

Let $\mathcal{R}_{1}=A_{1}+B_{1} D_{t}$ and $\mathcal{R}_{2}=A_{2}+B_{2} D_{t}$ be two scalar formal recursion operators. Then the product $\mathcal{R}_{3}=\mathcal{R}_{1} \mathcal{R}_{2}$, in which $D_{t}^{2}$ is replaced by $\left(M+N D_{t}\right)$ is also a scalar formal recursion operator whose components are given by (16).

An operator $\mathcal{S}=P+Q D_{t}$ is said to be implectic if

$$
\mathcal{F}^{*} S+\overline{\mathcal{S}} \mathcal{F}=0, \quad \overline{\mathcal{S}}=\bar{P}+\bar{Q} D_{t}
$$

Here and in the sequel the superscript $*$ denotes the adjoint operator. If $\mathcal{S}$ can be applied to symmetries, then it maps symmetries to cosymmetries. In the symmetry approach $P$ and $Q$ are supposed to be formal non-commutative series with respect to $D_{x}$.

The operator equations of components of the formal implectic operator $\mathcal{S}=P+Q D_{t}$ have the following form

$$
P_{t t}+U^{*} P_{t}+2 Q_{t} V+Q V_{t}=V^{*} P-P V-\left(Q U+U^{*} Q\right) V-U_{t}^{*} P
$$




$$
Q_{t t}+2 P_{t}+2 Q_{t} U+U^{*} Q_{t}=V^{*} Q-Q V-\left(Q U+U^{*} Q\right) U-\left(P U+U^{*} P\right)-\left(U_{t}^{*} Q+Q U_{t}\right) .
$$

The linearization $P+Q D_{t}$ of a variational derivative of any conserved density for equation (4) satisfies equations (18), (19) up to a "small" rest (see [11]).

Let us consider equations of the form (1). It follows from formulas (18), (19) that equation (1) has no higher conservation laws. Moreover, it is easy to prove that the density of any conservation law, up to total derivatives, is of the form

$$
\rho=r_{1}\left(q, q_{x}\right) q_{t}+r_{2}\left(q, q_{x}\right) .
$$

All statements presented below can be easily reformulated for general equations (4).

Theorem 1. If equation (1) possesses an infinite sequence of higher symmetries of the form

$$
q_{\tau_{i}}=G_{i}\left(q, q_{1}, q_{2}, \ldots, q_{r_{i}}, q_{t}, q_{t 1}, q_{t 2}, \ldots, q_{t s_{i}}\right)
$$

then there exists a formal recursion operator of the form

$$
\mathcal{R}=\left(a_{0}+a_{-1} D^{-1}+\ldots\right)+\left(D^{-1}+b_{-2} D^{-2}+\ldots\right) D_{t},
$$

where $a_{i}, b_{i}$ are some functions of the variables (8).

For scalar evolution equations one can use (see $[3,5,10])$ the residues of powers of the formal recursion operator to derive the canonical conservation laws (10). Unfortunately for equations (1) this technique does not work and we present a different way (cf.[12]) to get necessary integrability conditions (10).

Let $\mathcal{R}$ be a formal recursion operator of the form (21). It is then possible to find an operator

$$
\mathcal{R}^{-1}=\left(\alpha_{-1} D^{-1}+\alpha_{-2} D^{-2} \ldots\right)+\left(D^{-2}+\beta_{-3} D^{-3}+\ldots\right) D_{t}
$$

such that $\mathcal{R} \mathcal{R}^{-1}=\mathcal{R}^{-1} \mathcal{R}=1$. Recall that we eliminate $D_{t}^{2}$ in virtue of $\mathcal{F}=0$ in the product of scalar recursion operators. The operator $\mathcal{R}^{-1}$ is uniquely defined.

Theorem 2. If $\mathcal{R}$ is a formal recursion operator of the form (21) for equation (1), then there is a unique representation of the total derivative operators $D_{x}$ and $D_{t}$ of the form

$$
D_{x}=\sum_{-\infty}^{2} \rho_{i} \mathcal{R}^{i}, \quad D_{t}=\sum_{-\infty}^{3} \sigma_{i} \mathcal{R}^{i} .
$$

Functions $\rho_{i}$ and $\sigma_{i}$ are densities and fluxes of some (maybe trivial) conservation laws (10) for equation (1).

The next formulas define five integrability conditions (10) for equations (1):

$$
\begin{gathered}
\rho_{1}=u_{1}, \\
\rho_{2}=v_{2}+\frac{2}{3} \sigma_{1}, \\
\rho_{3}=6 \sigma_{2}-u_{1} \sigma_{1}+9 u_{0}-3 u_{1} v_{2}-\frac{1}{3} u_{1}^{3}, \\
\rho_{4}=6 \sigma_{3}-9 u_{1} \sigma_{2}+3 \sigma_{1}^{2}+27 u_{0} u_{1}-u_{1}^{4}+81 v_{1}-9 u_{1}^{2} v_{2}-27 v_{2}^{2} . \\
\rho_{5}=2 \sigma_{4}+18 \sigma_{1} \sigma_{2}-27\left(\sigma_{1}\right)_{t}-3 \sigma_{1}^{2} u_{1}-3 \sigma_{3} u_{1}-9 \sigma_{1} u_{1} v_{2}-\sigma_{1} u_{1}^{3}+27 \sigma_{1} u_{0} .
\end{gathered}
$$

The conditions mean that $\rho_{i}$ are densities of local conservation laws for equation (1). In other words, for any $\rho_{i}$ there exists a corresponding function $\sigma_{i}$ depending on variables (8). 


\subsection{List of integrable equations.}

Lemma 1. If equation (1) satisfies conditions (10) with $i=1,2,3,4$ then it is of the form

$$
\begin{aligned}
q_{t t}= & q_{x x x}+\left(A_{1} q_{t}+A_{2}\right) q_{t x}+A_{3} q_{x x}^{2}+\left(A_{4} q_{t}^{2}+A_{5} q_{t}+A_{6}\right) q_{x x}+ \\
& +A_{7} q_{t}^{4}+A_{8} q_{t}^{3}+A_{9} q_{t}^{2}+A_{10} q_{t}+A_{11}
\end{aligned}
$$

where the functions $A_{i}$ depend on $q$ and $q_{x}$ only.

It is easy to verify that the class of equations (24) is invariant with respect to point transformations $q \rightarrow \varphi(q)$. Moreover, if all functions $A_{i}$ do not depend on $q$, then the shifts of the form $q \rightarrow q+\lambda_{1} x+\lambda_{2} t$, where $\lambda_{i}$ are arbitrary constants, are also allowed.

Theorem 3. Up to the transformations described above, any nonlinear equation (1) satisfying integrability conditions with $i=1,2, \ldots, 7$, coincides with the equation

$$
q_{t t}=q_{x x x}+\left(3 q_{x}+k\right) q_{x t}+\left(q_{t}-3 q_{x}^{2}-2 k q_{x}+6 \mathcal{P}\right) q_{x x}-2 \mathcal{P}^{\prime} q_{t}+6 \mathcal{P}^{\prime} q_{x}^{2}+\left(\mathcal{P}^{\prime \prime}+k \mathcal{P}^{\prime}\right) q_{x}
$$

where $\mathcal{P}(q)$ is any solution of equation of the form

$$
\mathcal{P}^{\prime 2}=8 \mathcal{P}^{3}+k^{2} \mathcal{P}^{2}+c_{1} \mathcal{P}+c_{0}
$$

or with the equation

$$
q_{t t}=q_{x x x}+\left(\frac{3 q_{t}}{q_{x}}+\frac{3}{2} X\right) q_{x t}-\frac{1}{q_{x}} q_{x x}^{2}-\left(\frac{2 q_{t}^{2}}{q_{x}^{2}}+\frac{3 q_{t}}{2 q_{x}} X\right) q_{x x}+c_{2}\left(q_{x} q_{t}+\frac{3}{2} q_{x}^{2} X\right),
$$

where $X(q)=c_{2} q+c_{1}$ and $c_{i}$ are arbitrary constants.

Remarks. Actually, any equation (27) can be reduced to the equation with $X(q)=$ const or to the equation with $X(q)=q$. The integrability conditions 6 and 7 have quite long expressions and we do not present them in the paper. We have only used these conditions to prove that any equation (1) for which all conservation laws (10) are trivial is equivalent to a linear one.

\subsection{Recursion operators.}

In this Section we present a closed form of recursion operators for models (25) and (27). The existence of these recursion operators implies the fact that all integrability conditions with $i \geq 1$ are fulfilled for these equations.

Let us consider equation (25). This equation has the only non-trivial conserved density given by

$$
\rho=q_{t}-q_{x}^{2}+2 \mathcal{P}(q)
$$

The simplest higher symmetries of (25) are

$$
q_{\tau}=q_{x x}+2 q_{x} q_{t}-q_{x}^{3}-k q_{x}^{2}+4 \mathcal{P} q_{x}
$$

and

$$
q_{\tau}=q_{x t}+q_{x} q_{x x}+q_{t}^{2}+\left(q_{x}^{2}+2 \mathcal{P}\right) q_{t}-q_{x}^{4}-k q_{x}^{3}+6 \mathcal{P} q_{x}^{2}+\left(\mathcal{P}^{\prime}+k \mathcal{P}\right) q_{x}
$$


They are generated by the following recursion operator

$$
\mathcal{R}=D+\left(q_{t}-2 q_{x}^{2}-k q_{x}+2 \mathcal{P}\right)+q_{x} D^{-1}\left(D_{t}+2 q_{x x}+2 \mathcal{P}^{\prime}\right)
$$

acting on the seed symmetries $q_{x}$ and $q_{t}$. A direct calculation shows that this recursion operator satisfies (14) and (15). In the degenerate case $\mathcal{P} \equiv 0, k=0$ we have $\mathcal{R}=\mathcal{R}_{0}^{2}$, where $\mathcal{R}_{0}$ is defined by (3). The operator $D_{t}+2 q_{x x}+2 \mathcal{P}^{\prime}$ corresponds to the variational derivative of the function (28) and therefore if we apply $\mathcal{R}$ to any local symmetry admitting the conservation law with density (28), the result should be local.

Another recursion operator for (25) has the form

$$
\mathcal{S}=D_{t}+\left(q_{x x}-q_{x}^{3}-k q_{x}^{2}+6 \mathcal{P} q_{x}+k \mathcal{P}+\mathcal{P}^{\prime}\right)+q_{t} D^{-1}\left(D_{t}+2 q_{x x}+2 \mathcal{P}^{\prime}\right)
$$

One can verify that

$$
\mathcal{S}^{2}=\mathcal{R}^{3}-k \mathcal{R} \mathcal{S}-\frac{c_{1}}{2} \mathcal{R}-c_{0}
$$

For equation (27) a recursion operator is given by

$$
\mathcal{R}=\left(\frac{q_{t}}{q_{x}}+\frac{1}{2} X\right)-q_{x} D^{-1}\left(\frac{1}{q_{x}} D_{t}+\frac{q_{x t}}{q_{x}^{2}}-\frac{2 q_{t} q_{x x}}{q_{x}^{3}}+\frac{c_{2}}{2}\right) .
$$

The non-trivial conserved density for this equation is given by

$$
\rho=\frac{q_{t}}{q_{x}}+\frac{1}{2} X(q)
$$

In the case $X(q)=0, c_{1}=0$ the nonlocal variable $Q=D_{x}^{-1}(\rho)$ satisfies equation (2).

\section{Linearization procedure.}

Each of equations (25) and (27) has only one non-trivial conserved local density. The Burgers equation $u_{t}=u_{x x}+2 u u_{x}$ possesses the same property: $D_{t}(u)=D_{x}\left(u_{x}+u^{2}\right)$ is the only conservation law for this equation. The crucial step in the linearization of the Burgers equation is to introduce the potential $W$ of this conservation law. By definition the variable $W$ satisfies conditions $W_{x}=u, \quad W_{t}=u_{x}+u^{2}$. It is easy to verify that the function $U=\exp (W)$ satisfies the heat equation $U_{t}=U_{x x}$.

For equations (25) and (27) the potentials of the conservation laws also play a key role in linearization. However the procedure of linearization is not so straightforward. To illustrate this procedure, let us consider the simplest version $X=0$ of equation (27). The potential of the conservation law for this equation satisfies the conditions

$$
W_{x}=\frac{q_{t}}{q_{x}}, \quad W_{t}=\frac{q_{x x}}{q_{x}}+\frac{q_{t}^{2}}{q_{x}^{2}} .
$$

A simple computation shows that the equation admits the following non-local symmetry $q_{y}=W$. Since

$$
D_{y}\left(\frac{q_{t}}{q_{x}}\right)=\frac{q_{x x}}{q_{x}^{2}}
$$


we have

$$
q_{y y}=W_{y}=-\frac{1}{q_{x}}, \quad q_{y y y}=\frac{q_{t}}{q_{x}^{3}}
$$

or

$$
q_{x}=-\frac{1}{q_{y y}}, \quad q_{t}=-\frac{q_{y y y}}{q_{y y}^{3}} .
$$

After the Legendre transformation

$$
y=U_{z}, \quad q=U-z U_{z}
$$

the latter pair of compatible equations becomes

$$
U_{x}=U_{z z}, \quad U_{t}=U_{z z z}
$$

For the more complicated equation

$$
q_{t t}=q_{x x x}+\frac{3 q_{t}}{q_{x}} q_{x t}-\frac{1}{q_{x}} q_{x x}^{2}-\frac{2 q_{t}^{2}}{q_{x}^{2}} q_{x x}+c\left(q_{x t}-\frac{q_{t}}{q_{x}} q_{x x}\right)
$$

corresponding to the case $X=$ const $\neq 0$ the same linearization scheme works also. The potential is defined by

$$
W_{x}=\frac{q_{t}}{q_{x}}, \quad W_{t}=\frac{q_{x x}}{q_{x}}+\frac{q_{t}^{2}}{q_{x}^{2}}+c \frac{q_{t}}{q_{x}},
$$

the non-local symmetry is given by $q_{y}=\exp (-c W)$. It is not hard to check that

$$
q_{x}=-c^{2} \frac{q_{y}^{2}}{q_{y y}}, \quad q_{t}=c^{3} \frac{q_{y}^{3} q_{y y y}-2 q_{y}^{2} q_{y y}^{2}}{q_{y y}^{3}} .
$$

After a contact transformation

$$
y=\frac{1}{2} \exp (-z)\left(U_{z}+U\right), \quad q=\frac{1}{2} \exp (z)\left(U_{z}-U\right)
$$

these equations become

$$
U_{x}=\frac{c^{2}}{2}\left(U_{z z}-U\right), \quad U_{t}=\frac{c^{3}}{4}\left(U_{z z z}+U_{z z}-U_{z}-U\right)
$$

The most non-trivial case is $X=q$. In this case the potential $W$ is defined by

$$
W_{x}=\frac{q_{t}}{q_{x}}+\frac{q}{2}, \quad W_{t}=\frac{q_{x x}}{q_{x}}+\frac{q_{t}^{2}}{q_{x}^{2}}+\frac{3 q_{t}}{2 q_{x}} q+\frac{3}{4} q^{2}
$$

but non-local symmetries of the form $q_{y}=F(q, W)$ do not exist. However there exists a new non-local conservation law with potential $Z$ defined by

$$
Z_{x}=q^{2}-2 W q_{x}, \quad Z_{t}=-\frac{1}{2} q^{3}-2 q_{x}-2 W q_{t} .
$$


Using these two potentials, we find a non-local symmetry $q_{y}=2 q \exp \left(-\frac{Z}{4}-\frac{q W}{2}\right)$. Expressing $q_{x}$ and $q_{t}$ in terms of the $y$-derivative, we get

$$
q_{x}=-\frac{q^{3} q_{y}^{2}}{4\left(q q_{y y}-2 q_{y}^{2}\right)}, \quad q_{t}=\frac{q^{4} q_{y}^{3}\left(q^{2} q_{y y y}-9 q q_{y} q_{y y}+12 q_{y}^{3}\right)}{8\left(q q_{y y}-2 q_{y}^{2}\right)^{3}} .
$$

After the contact transformation $y=z+\frac{U}{U_{z}}, q=-\frac{U_{z}}{U^{2}}$ we do not obtain linear equations but

$$
U_{x}=\frac{1}{4} D_{z}\left(\frac{U_{z}}{U^{2}}\right), \quad U_{t}=-\frac{1}{8} D_{z}\left(\frac{U_{z z}}{U^{3}}-\frac{3 U_{z}^{2}}{U^{4}}\right) .
$$

To linearize the latter system one can introduce the potential $Y$ such that $Y_{z}=U, Y_{x}=$ $\frac{U_{z}}{4 U^{2}}$ and after that make a point transformation $Y \leftrightarrow z$.

Equation (25) can be linearized as follows. It is easy to verify that it has a non-local symmetry $q_{y}=A(q) \exp (-W)$, where

$$
W_{x}=q_{t}-q_{x}^{2}+2 \mathcal{P}, \quad W_{t}=q_{x x}-q_{x}^{3}+q_{t} q_{x}+k\left(q_{t}-q_{x}^{2}+\mathcal{P}\right)+6 \mathcal{P} q_{x}+\mathcal{P}^{\prime}+w
$$

and

$$
B^{\prime 2}=B^{4}+\frac{k^{2}}{2} B^{2}+8 w B+b_{0}, \quad B=-\frac{A^{\prime}}{A}+\frac{k}{2} .
$$

It can be checked that

$$
\begin{gathered}
q_{x}=\frac{q_{y y}}{q_{y}^{2}}+2 B(q) \\
q_{t}=-\frac{q_{y y y}}{q_{y}^{3}}+3 \frac{q_{y y}^{2}}{q_{y}^{4}}+3 \frac{q_{y y}}{q_{y}^{2}} B(q)+\frac{k}{2}\left(\frac{q_{y y}}{q_{y}^{2}}+2 B(q)\right)-\frac{3}{2}\left(B^{\prime}(q)-B(q)^{2}\right)-\frac{k^{2}}{8} .
\end{gathered}
$$

Function $\mathcal{P}$ from equation (25) is given by

$$
\mathcal{P}=-\frac{1}{4} B^{\prime}+\frac{1}{4} B^{2}-\frac{k^{2}}{48}
$$

where the parameters of the elliptic functions $\mathcal{P}$ and $B$ are related by

$$
c_{1}=\frac{k^{4}-16 b_{0}}{32}, \quad c_{0}=w^{2} .
$$

After change of variables $z=q, u=y$ equations (31), (32) take the following linear form

$$
\begin{gathered}
u_{x}=u_{z z}-2 B(z) u_{z}, \\
u_{t}=-u_{z z z}+3 B(z) u_{z z}+\frac{3}{2}\left(B^{\prime}(z)-B(z)^{2}\right) u_{z}+\frac{k}{2}\left(u_{z z}-2 B(z) u_{z}\right)+\frac{k^{2}}{8} u_{z} .
\end{gathered}
$$

We see that in all cases there exists a non-local symmetry $q_{y}=G$ depending on the potentials such that $q_{x}$ and $q_{t}$ can be expressed in terms of $y$-derivatives by formulas (7). Using this parametrization, one can construct particular solutions of equations (25) and (27). 
A parametrization of such kind arises not only for linearizable equations but also for equations of KdV-type and associated linear spectral problems. For example, let us consider the spectral problem

$$
\Psi_{x x}=\left(\lambda^{3}+u_{1} \lambda^{2}+u_{2} \lambda+u_{3}\right) \Psi
$$

For a non-local symmetry of this linear equation one can take $($ see $[9,1])$

$$
\bar{\Psi}_{y y}=\frac{\lambda}{a^{2}} \bar{\Psi}, \quad \bar{\Psi}=\frac{1}{\sqrt{a}} \Psi
$$

Then

$$
u_{1}=\frac{1}{4} a_{y}^{2}-\frac{1}{2} a a_{y y}, \quad u_{2}=-a(\log (a))_{x y}, \quad u_{3}=-\frac{a_{x x}}{2 a}+\frac{3 a_{x}^{2}}{4 a^{2}},
$$

where $a(x, y)$ satisfies the Harry-Dym equation

$$
a_{x}=a^{3} a_{y y y} .
$$

At least on the local level the general solution of the latter equation depends on three arbitrary functions $a_{0}(x)=a(x, 0), a_{1}(x)=a_{y}(x .0), a_{2}(x)=a_{y y}(x, 0)$ of $x$ and therefore this parametrization provides a generic potential in (33). Multi-phase solutions of the Harry-Dym equation lead to special potentials of the spectral problem (33).

\section{References}

[1] L. Martínez Alonso and A.B. Shabat, Towards a theory of differential constraints of a hydrodynamic hierarchy, to appear in J. Non. Math. Phys.

[2] Ibragimov N. Kh. and Shabat A. B., Infinite Lie-Bäcklund algebras, Functional Anal. and Appl., 14(4), 79-80, 1980. [in Russian]

[3] Sokolov V.V., Shabat A.B., Classification of Integrable Evolution Equations, Soviet Scientific Reviews, Section C, 4, 221-280, 1984.

[4] Drinfeld V.G., Svinolupov S.I. and Sokolov, V.V., Classification of fifth order evolution equations with infinite series of conservation laws, Doklady of Ukrainian Akademy, Section A, 10, 7-10, 1985.

[5] Mikhailov A. V., Shabat A. B., and Yamilov R. I., The symmetry approach to the classification of non-linear equations. Complete lists of integrable systems, Russian Math. Surveys, 42(4), 1-63, 1987.

[6] Svinolupov S.I., Generalized Schrödinger equations and Jordan pairs, Comm. in Math. Physics, 143(1), 559-575, 1992.

[7] Svinolupov S.I., Jordan algebras and generalized Korteweg-de Vries equations, Theor. and Math. Phys., 87(3), 391-403, 1991. 
[8] Svinolupov S. I., Second order evolution equations possessing symmetries, Russian Math. Surveys., 40(5), 263-264, 1985.

[9] L. Martínez Alonso and A.B. Shabat, Energy dependent potentials revisited. A universal hierarchy of hydrodynamic type, nlin. SI/0202008.

[10] Mikhailov A. V., Sokolov V.V., Shabat A.B., The symmetry approach to classification of integrable equations, in "What is Integrability?" (V.E. Zakharov ed.), Springer series in Nonlinear Dynamics, 115-184, 1991.

[11] Svinolupov S. I. and Sokolov V. V., Evolution equations with nontrivial conservation laws, Functional Anal. and Appl., 16(4), 86-87, 1982.

[12] Chen H. H., Lee Y. C., Liu C. S., Integrability of nonlinear Hamiltonian systems by inverse scattering method, Physica Scripta, 20(3/4), 490-492, 1979. 